Archivist

\title{
Avoiding respiratory complications in paediatric anaesthesia
}

Although there are guidelines for the management of anaesthesia, there is room for improvement in practice, especially for the prevention of respiratory complications in children. A study in Perth, Australia (Britta S von Ungern-Sternberg and colleagues. Lancet 2010;376:773-83; see also Comment, ibid: 745-6) has pointed the way to better practice.

The study included all 10496 children who underwent general anaesthesia for any reason at the Princess Margaret Hospital for Children in Perth between 1 February 2007 and 31 January 2008. Anaesthetists completed a questionnaire based on the International Study Group for Asthma and Allergies in Childhood questionnaire and recorded other relevant information on the day of anaesthesia. A total of 9297 questionnaires were analysed. The mean age of the children was 6.2 years; $20 \%$ were infants and $14 \%$ teenagers; $60 \%$ were boys. Periprocedural adverse respiratory events occurred in 1392 (15\%) of the 9297 children. The most common problem was oxygen desaturation ( $10 \%$ of the children), followed by coughing $(7 \%)$, airway obstruction (4\%), laryngospasm (4\%), bronchospasm (2\%) and stridor (1\%). The procedure was elective in $65 \%$ of children and urgent in $35 \%$. Induction of anaesthesia was with intravenous propofol in $61 \%$ of cases and inhaled sevoflurane in 39\%. Maintenance of anaesthesia was with an inhaled drug in $86 \%$ and with propofol in $14 \%$. A positive respiratory history (nocturnal dry cough, wheezing during exercise, $>3$ episodes of wheezing in the past 12 months, or past or present eczema) increased the risk of bronchospasm by a factor of 8.5 , laryngospasm by a factor of 4.1 , and perioperative cough, desaturation or airway obstruction by a factor of 3.1. An upper respiratory tract infection either present or within the last 2 weeks increased the risk of periprocedural respiratory complications 2.3 -fold, whereas an upper respiratory tract infection 2-4 weeks previously was associated with a significant $34 \%$ reduction in the risk. The risk was increased significantly if two or more family members had asthma or atopy, or smoked. Intravenous induction of anaesthesia was associated with less risk than inhalational induction, but inhalational maintenance was associated with less risk than intravenous maintenance. Other factors associated with less risk were use of a face mask rather than tracheal intubation and management by a specialist paediatric anaesthetist rather than a registrar.

It should be possible to anticipate many respiratory problems occurring during or after paediatric anaesthesia and take steps to avoid them.

Provenance and peer review Not commissioned; not externally peer reviewed.

Arch Dis Child 2011;96:29. doi:10.1136/adc.2010.206730 